Fungos Endofíticos: Uma Fonte Inexplorada e Sustentável de Novos e Bioativos Produtos Naturais

\author{
Chapla, V. M.; Biasetto, C. R.; Araujo, A. R.
}

Rev. Virtual Quim., 2013, 5 (3), 421-437. Data de publicação na Web: 11 de novembro de 2012

\author{
http://www.uff.br/rvq
}

\title{
Endophytic Fungi: An Unexplored and Sustainable Source of New and Bioactive Natural Products
}

\begin{abstract}
Endophytic fungi are a rich source of new and biologically active natural products. They colonize a relatively unexplored ecological habitat and their secondary metabolism is particularly active, presumably due to metabolic interactions with their hosts. In the course of our continuing investigations for new and bioactive compounds from endophytic fungi from brazilian flora Alibertia macrophylla, Caseria sylvestris, Ocotea corymbosa, Cassia spectabilis, Piper aduncum, Cryptocaria mandioccana, Xylopia aromatica and Palicourea marcgravii were investigated. Forty two natural products were isolated and their structures were established on the basis of comprehensive spectral analysis, mainly using $1 \mathrm{D}$ and 2D NMR experiments. The compounds were tested in their antifungal, antioxidant, anticholinesterasic and anticancer activities.
\end{abstract}

Keywords: Endophytic fungi; fungal metabolites; anticholinesterasic; antifungal; anticancer activities.

\section{Resumo}

Fungos endofíticos representam uma fonte rica de produtos naturais novos e bioativos. Eles colonizam um habitat ecológico praticamente inexplorado e seus metabólitos secundários são particularmente ativos, possivelmente devido às interações metabólicas com seus hospedeiros. No decorrer de nossas pesquisas na investigação de novos e bioativos compostos de fungos endofíticos da flora brasileira, foram estudas as espécies Alibertia macrophylla, Caseria sylvestris, Ocotea corymbosa, Senna spectabilis, Piper aduncum, Cryptocaria mandioccana, Xylopia aromatica e Palicourea marcgravii. Quarenta e dois produtos naturais foram obtidos e suas estruturas determinadas com base nas análises dos dados espectrais de RMN 1D e 2D. Os compostos foram submetidos à avaliação das atividades antifúngica, antioxidante, anticolinesterásica e anticancerígena.

Palavras-chave: Fungos endofíticos; metabólitos fúngicos; atividades anticolinesterásica; antifúngica; anticancerígena.

\footnotetext{
* Universidade Estadual Paulista "Júlio de Mesquita Filho", Núcleo de Bioensaios, Biossíntese e Ecofisiologia de Produtos Naturais (NuBBE), Instituto de Química, Rua Francisco Degni, 55, CEP 14800 900". Araraquara-SP, Brasil.

Maraujoar@iq.unesp.br DOI: $\underline{10.5935 / 1984-6835.20130036}$
} 


\section{Fungos Endofíticos: Uma Fonte Inexplorada e Sustentável de Novos e Bioativos Produtos Naturais \\ Vanessa M. Chapla, Carolina R. Biasetto, Angela R. Araujo*}

Universidade Estadual Paulista "Júlio de Mesquita Filho", Núcleo de Bioensaios, Biossíntese e Ecofisiologia de Produtos Naturais (NuBBE), Instituto de Química, Rua Francisco Degni, 55, CEP 14800-900". Araraquara-SP, Brasil.

* araujoar@iq.unesp.br

Recebido em 25 de maio de 2012. Aceito para publicação em 4 de novembro de 2012

\section{Introdução}

1.1. Produtos Naturais como fonte de medicamentos

1.2. Fungos endofíticos

1.3. Colonização de espécies vegetais por endófitos

1.4. Interação com o hospedeiro

1.5. Produtos naturais obtidos de fungos endofíticos

\section{O estudo de fungos endofíticos no NuBBE}

2.1. Citocalasinas

2.2. Isocumarinas

2.3. Sesquiterpenos presilfiperfolano e eremofilano

2.4. Sesquiterpenos cadalenos e calamenenos

2.5. Benzopiranos

2.6. Tetralonas

2.7. Outras substâncias

\section{Conclusões}

\section{Introdução}

1.1. Produtos Naturais como fonte de medicamentos

Por milhares de anos a medicina e os produtos naturais estão intimamente ligados através da utilização de medicamentos populares e o uso de venenos naturais. ${ }^{1}$
Estudos químicos, farmacológicos e clínicos dos medicamentos populares, derivados principalmente de plantas, ${ }^{1-4}$ foram a base de uma grande parte dos medicamentos tais como, por exemplo, a Aspirina ${ }^{\circledR}$ (ácido acetil salićlico) derivada da salicina, encontrada em diversas plantas do gênero Salix, ${ }^{4}$ digitoxina, morfina, quinina e pilocarpina. ${ }^{1}$

A descoberta acidental da penicilina, por Fleming em 1928, isolada do fungo Penicillium notatum, deu início aos estudos 
investigativos dos fungos, uma das fontes mais prolíficas de produtos naturais. ${ }^{2,4} \mathrm{~A}$ partir destes organismos, ocorreu um grande avanço nas áreas dos antibióticos, imunossupressores e medicamentos antineoplásicos. ${ }^{2}$ Alguns fármacos bem conhecidos e derivados de fungos são os antibióticos da classe das penicilinas e cefalosporinas, os redutores de colesterol como a mevastatina e lovastatina, imunossupressores como a ciclosporina e rapamicina, entre outros. ${ }^{5,6}$ Pesquisas mostram o enorme potencial dos fungos em produzir diversas classes de substâncias que podem ser utilizadas tanto na medicina como na agricultura. ${ }^{6,7}$

Apesar da concorrência com outros métodos de descoberta de novos medicamentos, os produtos naturais ainda desempenham um papel significativo na descoberta de novas drogas e no desenvolvimento desta área. ${ }^{1,8}$ Isto foi mostrado por Newman e Cragg $^{9}$ que analisaram os agentes terapêuticos aprovados, em todo o mundo, nos últimos 30 anos (1981 a 2010). Das novas entidades químicas aprovadas, $29 \%$ foram obtidas de origem natural. Dos medicamentos anticancerígenos, $48,6 \%$ são obtidos de produtos naturais ou diretamente derivados destes, e dos medicamentos aprovados para tratamento de infecções contra bactérias, fungos, parasitas e vírus, 33,7\% são obtidos a partir de fontes naturais. Esta constatação mostra como os produtos naturais são uma fonte expressiva para a obtenção de medicamentos, e que estes foram e ainda são valorizados como fonte de protótipos para a descoberta de novos fármacos. ${ }^{10}$

Os micro-organismos (fungos, bactérias, actinomicetos, entre outros) desempenham um papel fundamental na produção de novos produtos naturais que podem ser utilizados na indústria farmacêutica (humana e animal), alimentícia e agrícola. Os fungos encontrados no interior de espécies vegetais, denominados fungos endofíticos ou fungos endófitos, apresentam um enorme potencial na produção de substâncias novas e bioativas.

\subsection{Fungos endofíticos}

O termo endófito originalmente descrito por De Bary em 1866, refere-se a qualquer micro-organismo que vive nos tecidos de plantas, distinguindo-se dos epifíticos que vivem na superficie. São encontradas diferentes definições de endófito na literatura, ${ }^{11}$ mas a definida por Bacon e Write amplamente aceita e utilizada, é que endófitos são micro-organismos que colonizam os tecidos internos das plantas sem causar prejuízos imediatos no hospedeiro. ${ }^{12}$

Os fungos endofíticos são um grupo diversificado de ascomicetos definidos por sua ocorrência assintomática nos tecidos vegetais. Eles ocorrem em todo o território terrestre, nas comunidades naturais $e$ antrópicas, colonizando plantas no Ártico, Antártica, solos geotérmicos, desertos, oceanos, florestas tropicais, mangues e florestas costeiras. ${ }^{13,14}$ Em quase todas as plantas vasculares, algas marinhas, musgos e samambaias, estudadas até o momento, foram encontradas bactérias e fungos endofíticos. Normalmente, centenas de espécies de endófitos podem ser isolados de uma única planta, sendo que pelo menos um é específico ao hospedeiro. ${ }^{15}$

\subsection{Colonização de espécies vegetais por endófitos}

A espécie hospedeira pode ser infectada pelos endófitos horizontalmente por lesões naturais, como estômatos ou crescimento das raízes, e artificiais, como injúrias causadas por práticas agrícolas. A infecção também pode ocorrer verticalmente pelas sementes do hospedeiro, neste caso, o endófito pode se instalar em uma planta por toda sua vida. ${ }^{16,17} \mathrm{O}$ modo com que o fungo infecta uma espécie vegetal pode alterar o tipo de interação endófito-hospedeiro. $\mathrm{Na}$ transmissão vertical é predominante a interação mutualística, enquanto que na transmissão horizontal (não-sistêmica) essa 
interação tende a ser antagonista. ${ }^{17}$ Uma vez no hospedeiro 0 endófito permanece, geralmente, em um estado latente por toda sua vida ou por um período prolongado, até que as condições ambientais lhe sejam favoráveis. Neste caso, o endófito pode assumir uma função patogênica. ${ }^{18}$

Fungos endofíticos podem ser detectados por exame microscópico direto dos tecidos vegetais, e isolados a partir do hospedeiro (Figura 1). ${ }^{19}$

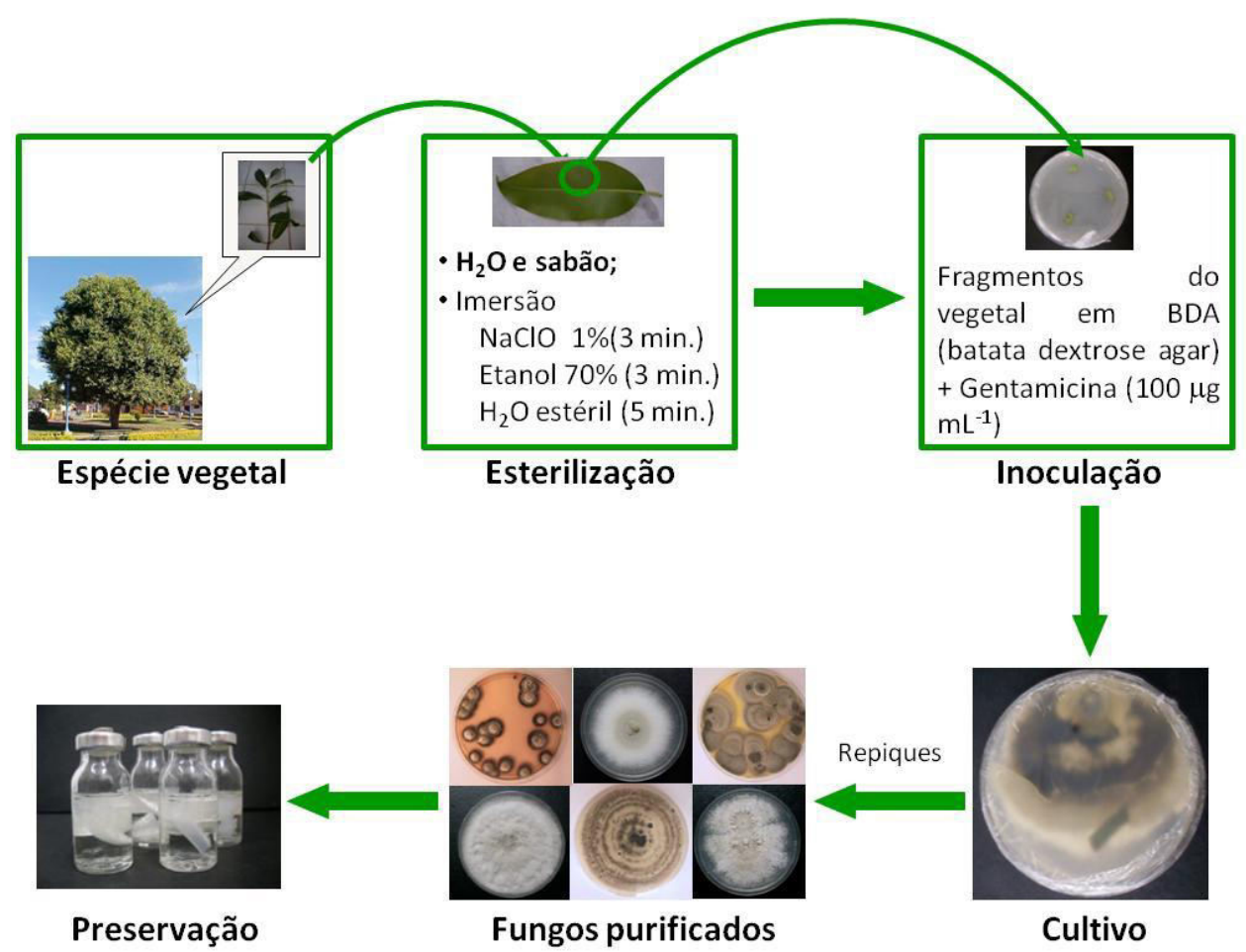

Figura 1. Metodologia de isolamento e purificação de fungos endofíticos ${ }^{20}$

\subsection{Interação com o hospedeiro}

Apesar de espécies dos gêneros Phomopsis, Phoma, Colletotrichum e Phyllosticta serem descritas colonizando diversas plantas, é conhecido que alguns endófitos são específicos ao hospedeiro e podem crescer em diferentes condições ecológicas e geográficas. ${ }^{13,17}$ A relação de um endófito com o hospedeiro pode variar de um hospedeiro para outro, e as interações entre eles ainda são pouco compreendidas. ${ }^{13,17,21}$ Esta associação sugere que estes micro-organismos coevoluiram com os seus hospedeiros, apresentando uma íntima relação mutualística, onde os endófitos recebem nutrientes e proteção enquanto a planta tem vantagens decorrentes dessa interação, como a maior resistência em ambientes com intenso estresse causado por fatores bióticos (insetos, herbívoros, nematoides parasitas e micro-organismos fitopatogênicos) ou abióticos $(\mathrm{pH}$, temperatura, estresse hídrico, ventos fortes, salinidade, etc. $)^{11,12,22,23 \text {, }}$

\subsubsection{Controle de insetos}

Alguns fungos endofíticos podem ser entomopatogênicos, como o fungo Beauveria bassiana, encontrado em milho, que protege o hospedeiro contra o ataque de insetos. Estudos pioneiros nos anos 80 mostraram que a presença de fungos endofíticos pode reduzir $o$ ataque de insetos à planta 
hospedeira, sendo descrito, a partir deste período, inúmeras espécies com potencial para o controle biológico de pragas. ${ }^{11,16,22}$ Posteriormente descobriu-se que os endófitos produzem toxinas ou substâncias que diminuem a atratividade da planta, aumentam a susceptibilidade do inseto a doenças ou inibem o seu desenvolvimento. ${ }^{22}$

\subsubsection{Controle de fitopatógenos}

Os micro-organismos endofíticos habitam um nicho ecológico semelhante aquele ocupado por fitopatógenos, podendo assim controlá-los por meio de competição por nutrientes, produção de substâncias antagônicas, parasitando o patógeno ou mesmo induzindo a planta a desenvolver resistência. ${ }^{22}$ A produção de compostos, como os antibióticos, sugere que os endófitos podem controlar doenças de plantas. ${ }^{13,22}$

Em alguns casos, nematoides podem ser controlados por endófitos, como o fungo Fusarium oxysporum que produz metabólitos contra o nematoide Meloidogyne incognita. Os fungos endofíticos produzem alcaloides que podem apresentar atividade inseticida e nematicida, protegendo a planta destes patógenos. ${ }^{11,16}$

1.4.3. Efeitos dos endófitos sobre os herbívoros

Os endófitos protegem as plantas contra o ataque de herbívoros. Algumas toxicoses induzidas em herbívoros domésticos, por ingestão de plantas, estão relacionadas com os micro-organismos endofíticos, principalmente os fungos. ${ }^{16}$

1.5. Produtos naturais obtidos de fungos endofíticos

Os micro-organismos endófitos associados a plantas representam uma fonte inexplorada de produtos naturais novos e bioativos, com mais de 20000 substâncias descritas ${ }^{23}$, sendo que destas $51 \%$ apresentam estruturas inéditas e $80 \%$ atividade biológica. ${ }^{24,25}$ Isto pode ser explicado pela teoria ecológica, que estabelece que esta produção metabólica depende do nicho ecológico no qual o microorganismo está inserido e das consequentes interações bióticas e abióticas. ${ }^{3,10}$ Estes relatos sugerem que a seleção do endófito para estudo, deve se realizada com espécies vegetais de diferentes biotas, principalmente as que enfrentam frequentes $e$ intensas interações no ambiente como plantas de regiões áridas, florestas tropicais, entre outras. $^{26}$

Os produtos naturais de fungos endofíticos apresentam um amplo espectro de atividades biológicas, sendo alguns exemplos, antimicrobiana (I-VIII), ${ }^{17,27}$ antiparasitária (VIII-XI), ${ }^{17,28}$ neuroprotetiva (XII), ${ }^{18}$ antioxidante (XIII-XIV), ${ }^{13}$ antidiabética $(\mathbf{X V}){ }^{29}$ propriedades imunossupressoras $(\mathbf{X V I}){ }^{3} \quad$ antiviral (XVII-XVIII), ${ }^{3,30}$ anticolinesterásica $\quad(\mathbf{X I X}),{ }^{17}$ antineoplásicos $(\mathbf{X X}-\mathbf{X X V I I})^{31,32}$ e citotóxica (XXVIII $)^{17,18}$ (Figura 2). Os metabólitos I a XXXI podem ser agrupados em várias classes, incluindo alcaloides, esteroides, terpenos, isocumarinas, quinonas, fenilpropanoides, lignanas, ácidos fenólicos, entre outros. ${ }^{31}$

Alguns estudos mostram que os fungos endofíticos são capazes de produzir um grande número de importantes metabólitos secundários bioativos, conhecidos apenas em plantas. ${ }^{6,13,17,18} \mathrm{Um}$ exemplo bem conhecido é a produção do Taxol $\AA$, um importante fármaco anticancerígeno, pelo fungo endofítico Taxomyces andreanae, isolado da planta Taxus brevifolia, que também produz esta substância. Outro importante anticancerígeno é a vincristina isolada da planta Catharanthus roseus, e recentemente isolada do fungo endofítico Fusarium oxysporum obtido da mesma planta. ${ }^{13}$ Podofilotoxina, também utilizada no tratamento de câncer, é encontrada em espécies vegetais do gênero Podophylum e também relatada nos endófitos Trametes hirsuta e Phialocephala fortinii. ${ }^{17}$ Existem 
outros exemplos de compostos de origem vegetal que também são produzidos por fungos que habitam os vegetais, ${ }^{14,17}$ sugerindo haver uma transposição de genes entre as plantas e os fungos em uma verdadeira engenharia genética in vivo. ${ }^{31}$

Das aproximadamente 300 mil espécies de plantas existentes, cada uma é hospedeira de pelo menos um micro-organismos endofítico e, poucas foram as espécies vegetais estudadas em relação a sua biologia endofítica. Consequentemente, é grande a oportunidade de descoberta de novos e interessantes micro-organismos endofíticos de plantas de diferentes ecossistemas. ${ }^{4,11,33}$

Estas evidências atraíram a atenção dos químicos de produtos naturais e de biólogos, conforme indicado pelo aumento constante de publicações dedicadas a este tema nos últimos anos (113 artigos científicos sobre metabólitos secundários de fungos endofíticos no período de 2008-2009, 69 de 2006-2007, 36 de 2004-2005, 14 de 20022003 e 18 de 2000-2001). ${ }^{18}$

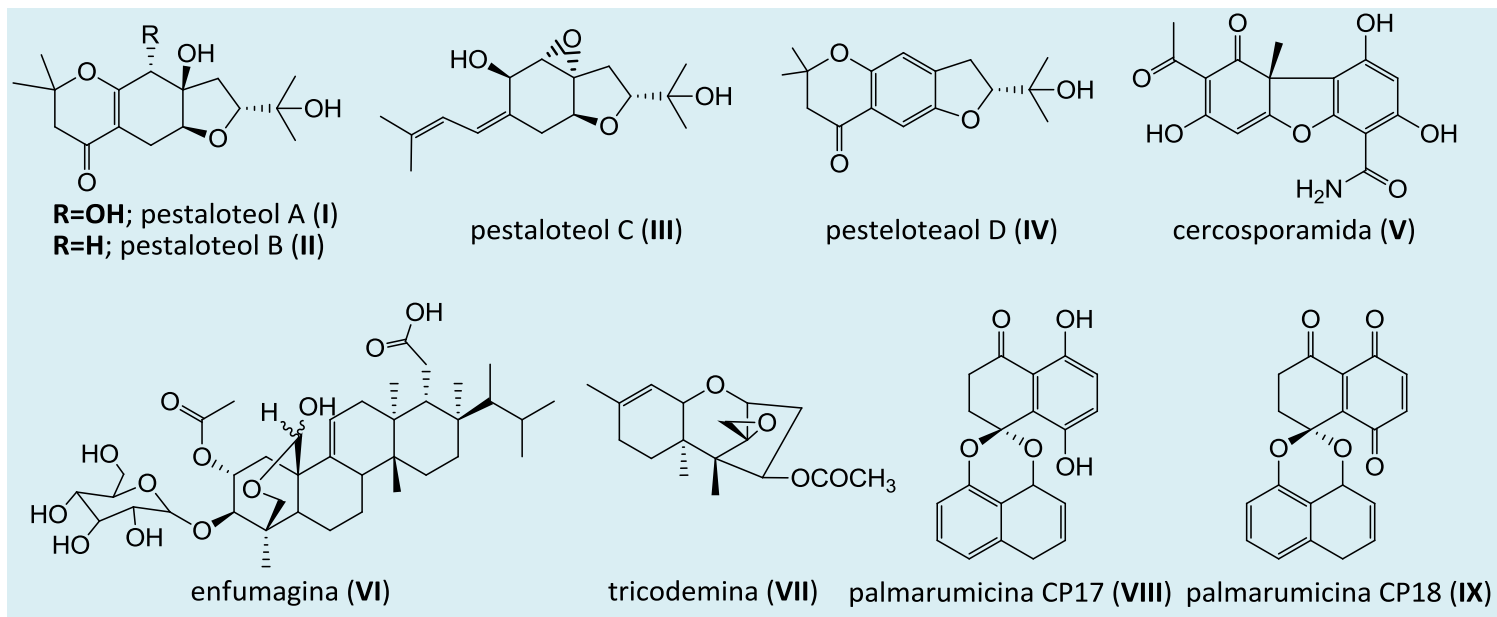

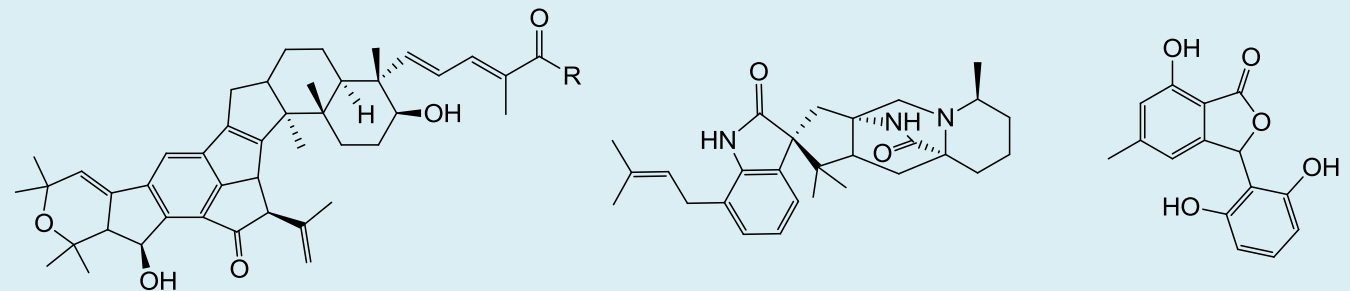

$\mathbf{R}=\mathbf{O H}$; ácido nodulispórico $\mathrm{A}(\mathbf{X}) \quad$ crisogenamida $\mathrm{A}(\mathbf{X I I}) \quad$ isopestacina (XIII) $\mathbf{R}=\mathbf{N H}_{2}$; terc-butil nodulisporamida $(\mathbf{X I})$

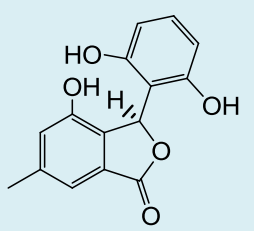

pestacina (XIV)

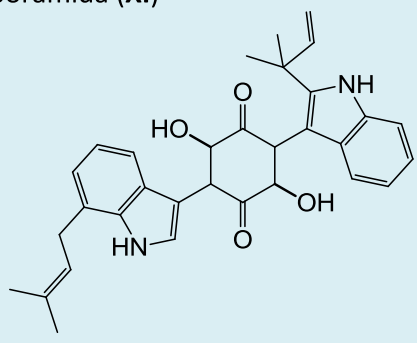

L $783.281(\mathrm{XV})$

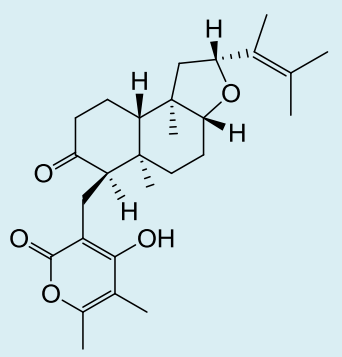

subglutinol A (XVI)

Figura 2. Substâncias bioativas produzidas por fungos endofíticos 


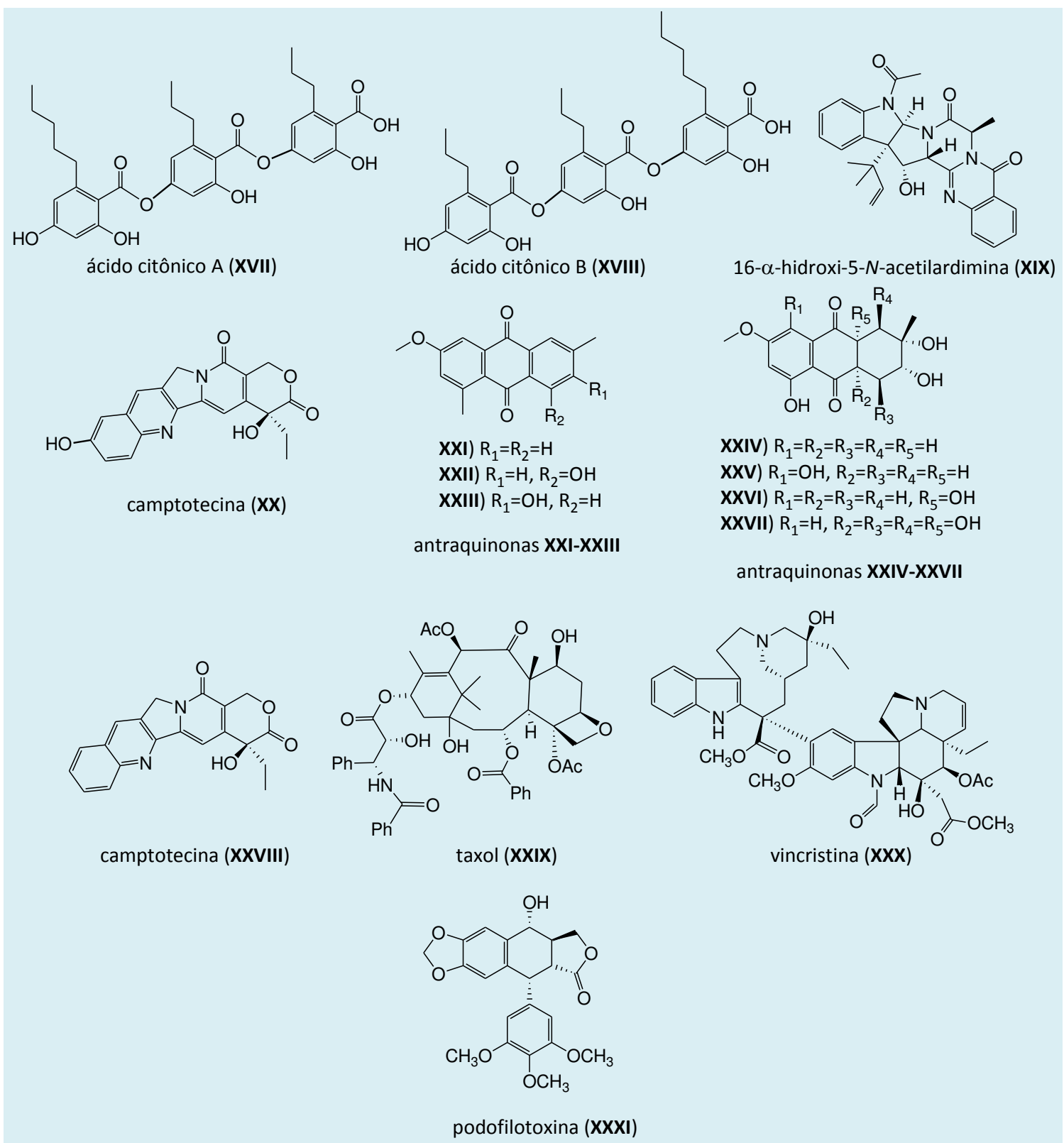

Figura 2. Continuação

\section{2. $O$ estudo de fungos endofíticos no NuBBE}

A continuidade dos esforços empregados para implantação e consolidação do estudo de micro-organismos endofíticos no Brasil, em desenvolvimento no NuBBE (Núcleo de Bioensaios, Biossíntese e Ecofisiologia Vegetal) do Instituto de Química da UNESP de Araraquara-SP, trouxe resultados interessantes e promissores, os quais alguns são abordados a seguir.
Neste trabalho, são apresentados os resultados obtidos dos estudos realizados com os endófitos associados às espécies vegetais: Alibertia macrophylla (Penicillium sp.1, Penicillium sp.2, Camarops sp., Penicillium sp.), Piper aduncum (Xylaria sp.1, Xylaria sp.2), Ocotea corymbosa (Curvularia sp.), Cassia spectabilis (Phomopsis cassiae), Cryptocaria mandioccana (Colletotrichum gloeosporioides), Xylopia aromatica (Periconia atropurpurea) e Palicourea marcgravii (Xylaria sp.).

Para o isolamento dos endófitos e para o estudo químico e biológico da microbiota 
endofítica associada, foram selecionadas espécies vegetais já estudadas por pesquisadores do NuBBE e que apresentaram substâncias potencialmente bioativas, com o objetivo de compreender-se a relação ecológica entre o endófito e a espécie hospedeira.

Das sete espécies vegetais selecionadas, 45 fungos endofíticos foram isolados $\mathrm{e}$ purificados. No entanto, neste trabalho, descreve-se a prospecção química e biológica de apenas onze endófitos. $O$ isolamento e a purificação dos endófitos a partir das espécies vegetais foram realizados de acordo com o fluxograma da Figura 1, e a metodologia geral para obtenção do extrato bruto e isolamento das substâncias é mostrada na Figura 3.

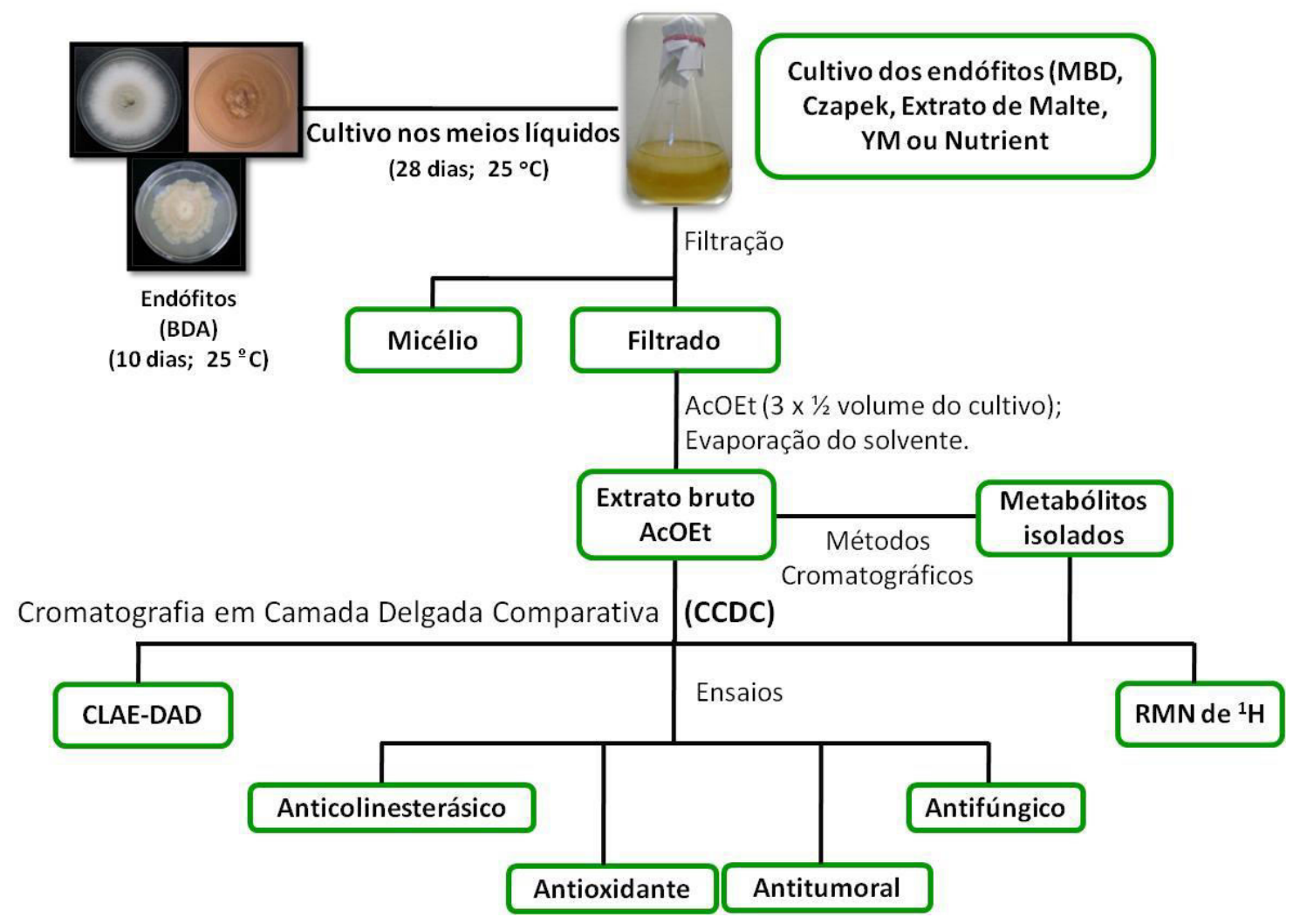

Figura 3. Metodologia de cultivo e obtenção dos extratos brutos para isolamento dos metabólitos produzidos pelos endófitos

\subsection{Citocalasinas}

Citocalasinas são terpenos produzidos por fungos e foram isoladas pela primeira vez em 1966. Existem mais de 60 citocalasinas conhecidas que foram isoladas de uma variedade de fungos, incluindo espécies de Helminthosporium sp., Phoma sp., Xylaria sp., Hypoxylon sp., e Chalara sp.. Estruturalmente são compostas de um anel isoindolona altamente substituído, com um grupo benzil na posição 3 e fundido com um anel macrocíclico de 11 a 14 membros. As citocalasinas exibem um amplo espectro de atividades, incluindo atividade antibiótica, antitumoral, inibidor de proteases de HIV, atividade fitotóxica, entre outras. São muito usadas em sondas biológicas (substâncias utilizadas em ensaios biológicos), mas sua aplicação terapêutica é limitada devido a sua toxicidade. $^{34}$ 
Dos endófitos Xylaria sp1 e Xylaria sp2 isolados de Piper aduncum e Senna spectabilis, respectivamente, foram isoladas as citocalasinas, 19,20-epoxicitocalasina (1) inédita, D (2), C (3), N (4), Q (5) e R (6) (Figura 4), todas apresentaram forte atividade contra a linhagem tumoral HeLa (tumor de cervix humano), com $\mathrm{Cl}_{50} 43,0 ; 1,0 ; 2,0 ; 1,0 ; 3,0 \mathrm{e}$ $3,0 \mu \mathrm{mol} \mathrm{L}^{-1}$, respectivamente. As citocalasinas 2-3 e 5-6 apresentaram atividade fraca contra a linhagem tumoral $\mathrm{CHO}$ (ovário de hamster Chinês). A forte atividade apresentada pelas citocalasinas 2-5 contra a linhagem cancerígena HeLa, e a baixa atividade contra a linhagem não cancerígena $\mathrm{CHO}$ evidenciam uma seletividade destas substâncias para as linhagens tumorais HeLa, sugerindo uma potencial utilização como droga anticancerígena. ${ }^{20}$

Do fungo Xylaria sp. isolado de Palicourea marcgravii foram isoladas as citocalasinas $D$ (7) e B (8) que apresentaram atividade contra os fungos fitopatogênicos Cladosporium cladosporioides e Cladosporium sphaerospermum a uma concentração de 10 e $25 \mu \mathrm{g} \mathrm{mL}^{-1}$, respectivamente. ${ }^{35}$

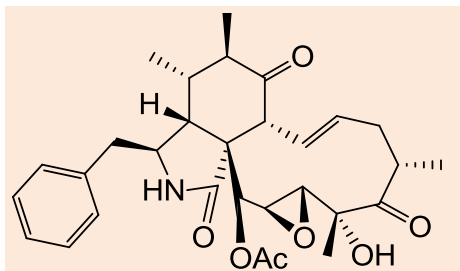

19,20-epoxicitocalasina (1)

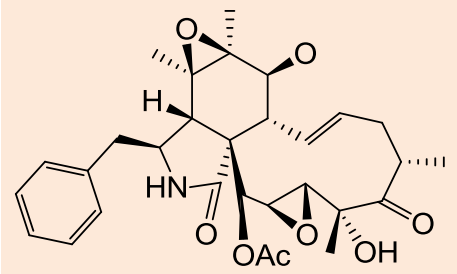

19,20-epoxicitocalasina N (4)

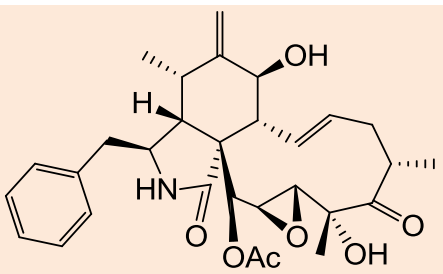

19,20-epoxicitocalasina D (2)

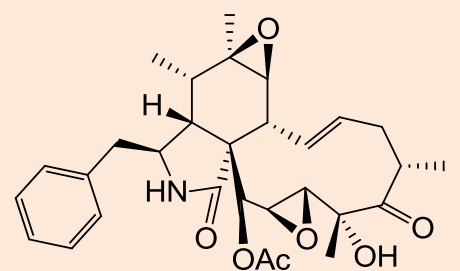

19,20-epoxicitocalasina Q (5)

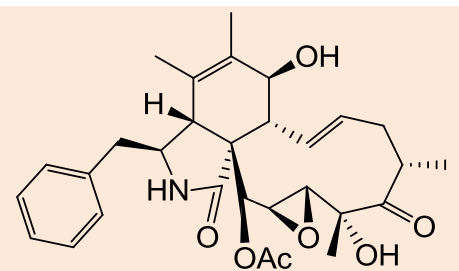

19,20-epoxicitocalasina C (3)

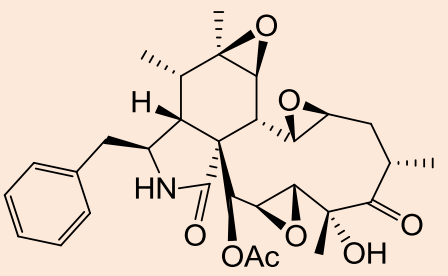

19,20-epoxicitocalasina R (6)

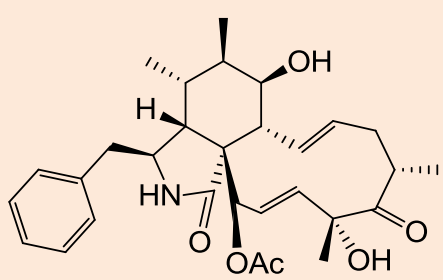

citocalasina $\mathrm{D}(\mathbf{7})$

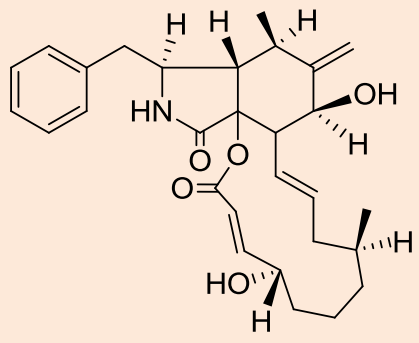

citocalasina B (8)

Figura 4. Citocalasinas produzidas pelos endófitos Xylaria sp. isolados de $P$. marcgravii

\subsection{Isocumarinas}

Isocumarinas são metabólitos secundários encontrados em uma grande variedade de organismos, como bactérias, fungos, liquens, entre outros. Esta classe de substâncias apresenta diversas atividades biológicas como inibidora de proteases, antimicrobiana, reguladoras de crescimento, antialérgica, antimalárica, etc. $^{36,38}$

Em nosso grupo de pesquisa, diversas isocumarinas foram isoladas e identificadas. ${ }^{37}$ Do endófito Penicillium sp.2, isolado de Alibertia macrophylla, foram isoladas as substâncias 4-hidroximeleina (9), 8metoximeleina (10) e 5-hidroximeleina (11) (Figura 5). A substância 9 mostrou moderada atividade contra os fungos fitopatogênicos $C$. 
cladosporioides e C. sphaerospermum com limite de deteç̧ão de 10,0 e 25,0 $\mu \mathrm{g}$, respectivamente. As substâncias $\mathbf{1 0}$ e $\mathbf{1 1}$ mostraram forte atividade com limite de detecção de 5,0 e 10,0 $\mu \mathrm{g}$ em C. cladosporioides e C. sphaerospermum, respectivamente. Adicionalmente, as substâncias 9, 10 e $\mathbf{1 1}$ inibiram a enzima acetilcolinesterase (AChE) com um limite de deteç̧ão de 3,$0 ; 10,0$ e $10,0 \mu \mathrm{g}$, respectivamente. $^{37}$

De Alibertia macrophylla e Piper aduncum foram isolados os endófitos Penicillium sp. e Xylaria sp. $\mathrm{O}$ extrato bruto do AcOEt obtido do cultivo em MBD (meio de batata dextrose) forneceu as isocumarinas $(3 R, 4 R)$-5-carboxi6-hidroxi-3-metil-3,4-di-hidroisocumarina (12) inédita, (3R) 7-hidroximeleina (13) e $(3 R, 4 R)$ 4,7-di-hidroximeleina (14). As substâncias 12-14 foram avaliadas quanto à atividade contra os fungos fitopatogênicos $C$. cladosporioides e C. sphaerospermum, e $\mathbf{1 2}$ apresentou forte atividade com um limite de detecção de 5,00 e 10,0 $\mu$ g, respectivamente. A substância 13 exibiu moderada atividade em $C$. cladosporioides e $C$. sphaerospermum, com limite de detecção de 10,0 e 25,0 $\mu \mathrm{g}$, respectivamente. ${ }^{38}$<smiles>CC1OC(=O)c2c(O)cccc2C1O</smiles><smiles>COc1cccc2c1C(=O)OC(C)C2</smiles><smiles>CC1Cc2c(O)ccc(O)c2C(=O)O1</smiles>

4-hidroximeleina (9)

8-metoximeleina (10) 5-hidroximeleina (11)<smiles>C[C@H]1OC(=O)c2ccc(O)c(C(=O)O)c2[C@H]1O</smiles>

(3R,4R)-5-carboxi-6-hidroxi-3-metil-3,4-di-hidroisocumarina (12)<smiles>C[C@H]1Cc2ccc(O)c(O)c2C(=O)O1</smiles><smiles>C[C@@H]1OC(=O)c2c(ccc(O)c2O)[C@H]1O</smiles>

(3R)-7-hidroximeleina (13) $\quad(3 R, 4 R)-4,7-$ di-hidroximeleina (14)

Figura 5. Isocumarinas produzidas por Xylaria sp. e Penicillium sp.

\subsection{Sesquiterpenos presilfiperfolano e eremofilano}

Sesquiterpenos eremofilanos são metabólitos secundários bem conhecidos em fungos e plantas superiores. Membros desta classe apresentam atividade fitotóxica, carcinostática, reguladora de crescimento de plantas, inibidora de enzimas, antibacteriana, citotóxica, entre outras. ${ }^{39,41}$

De Piper aduncum foi isolado o endófito Xylaria sp., que forneceu, após fracionamento do extrato bruto de AcOEt, os sesquiterpenos presilfiperfolanos, ácido 9,15di-hidroxipresilfiperfolano-4-oico (15), ácido 15-acetoxi-9-hidroxipresilfiperfolano 4-oico (16) e os eremofilanos faseolinona (17) e fomenona (18) (Figura 6). Os compostos 15 e 16 mostraram-se inativos nos ensaios para avaliação de atividade citotóxica em $\mathrm{CHO}$. Nos ensaios com os fitopatógenos $C$. cladosporioides e C. sphaerospermum, 16 mostrou atividade com limite de detecção de 10,0 $\mu \mathrm{g}$. Somente a substância 18 mostrou 80 e $50 \%$ de viabilidade celular em $\mathrm{CHO}$ a 20 e 
$200 \mu \mathrm{mol} \mathrm{L}^{-1}$, respectivamente. $^{40}$

Camarops sp., um endófito de Alibertia macrophylla, foi submetido ao cultivo em milho e forneceu após fracionamento cromatográfico do extrato bruto de AcOEt, os sesquiterpenos eremofilanos Xylarenona A (19) e as substâncias inéditas Xylarenona $C$ (20), Xylarenona D (21) e Xylarenona E (22). ${ }^{41}$

As substâncias 19-22 foram avaliadas in vitro quanto à atividade inibitória de proteases utilizando as enzimas subtilisina e pepsina. A substância $\mathbf{2 0}$ mostrou potente atividade inibitória nas enzimas pepsina com $\mathrm{Cl}_{50} 0,288 \mu \mathrm{mol} \mathrm{L}^{-1}$ e subtilisina com $\mathrm{Cl}_{50} 0,462$ $\mu \mathrm{mol} \mathrm{\textrm {L } ^ { - 1 }}$. O composto 19 também mostrou significante atividade inibitória da pepsina com $\mathrm{Cl}_{50} 2,43 \mu \mathrm{mol} \mathrm{L}^{-1}$. As substâncias 19, 21 e 22 não mostraram atividade inibitória na subtilisina nas concentrações testadas. Estes resultados sugerem que as hidroxilas da cadeia alifática lateral das substâncias $\mathbf{2 1}$ e 22 não sejam responsáveis pela atividade inibitória da protease. ${ }^{41}$

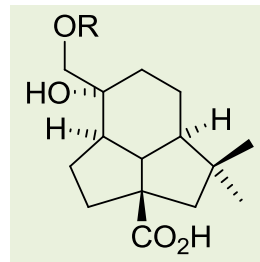

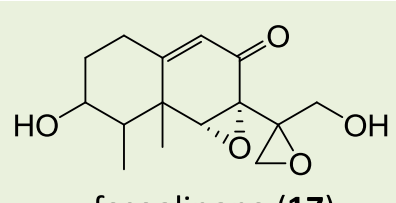

faseolinona (17)<smiles>C=C(CO)C1C(=O)C=C2CC[C@@H](O)[C@H](C)[C@@]2(C)C1=O</smiles>

fomenona (18)

$\mathrm{R}=\mathrm{H}$; ácido 9,15-di-hidroxipresilfiperfolano-4-oico (15)

$\mathrm{R}=\mathrm{COCH}_{3}$; ácido 15-acetoxi-9-hidroxipresilfiperfolano-4-oico (16)<smiles>C=C(CO)C1C(=O)C=C2C(O)CCC(C)C2(C)C1=O</smiles>

xylarenona A (19)<smiles>C=C(CO)[C@H]1C(=O)C=C2[C@@H](OC(=O)C(C)CC(C)CC(C)CC)CCC(C)[C@]2(C)C1C</smiles>

xylarenona C (20)<smiles>C=C(CO)[C@H]1C(=O)C=C2[C@@H](OC(=O)C(CO)(CO)CC(C)CC(C)CC)CCC(C)[C@]2(C)C1C</smiles>

xylarenona D (21)

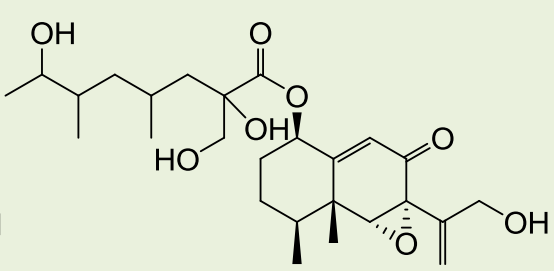

xylarenona $\mathrm{E}(\mathbf{2 2})$

Figura 6. Sesquiterpenos eremofilanos e presilfiperfolano isolados de Xylaria sp. e Camarops sp.

2.4. Sesquiterpenos cadalenos e citotóxica, antimicrobiana, entre outras. ${ }^{42,43}$ calamenenos

De Cassia spectabilis foram isolados sete endófitos, dentre estes Phomopsis cassiae foi

Os sesquiterpenos cadinanos são metabólitos secundários produzidos principalmente por fungos e corais, e apresentam atividades nematicida, cultivado em MBD e o seu extrato bruto de AcOEt, forneceu após fracionamentos cromatográficos sucessivos, os sesquiterpenos calamenenos e cadalenos inéditos (-)-(7S,9S,10S)-3,9,12-tri- 
hidroxicalameneno (23) e (+)-(7S,9R,10S)3,9,12-tri-hidroxicalameneno (24) (7S,10S)-3,12-di-hidroxicalameneno 3,12-di-hidroxicadaleno (26) e 3,11,12-trihidroxicadaleno (27) (Figura 7). As substâncias 23-27 foram avaliadas nos ensaios in vitro contra os fungos fitopatogênicos $C$. cladosporioides e $C$. sphaerospermum e $\mathbf{2 4}$ exibiu uma potente atividade com um limite de detecção de 5,0 $\mu \mathrm{g}$, comparável com o padrão nistatina. Nos ensaios para a avaliação da citotoxicidade utilizando a linhagem de tumor cervical (HeLa), as substâncias $\mathbf{2 5 - 2 7}$ exibiram moderada e fraca citotoxicidade com $\mathrm{Cl}_{50} 20$, 100 e $110 \mu \mathrm{mol} \mathrm{L}^{-1}$, respectivamente. ${ }^{43}$<smiles>Cc1cc2c(cc1O)[C@@H](C)[C@H](O)C[C@H]2C(C)CO</smiles>

(-)-(7S, 9S, 10S)-3,9,12-tri-hidroxicalameneno (23)<smiles>Cc1cc2c(cc1O)[C@H](C)CC[C@@H]2C(C)CO</smiles>

(+)-(7S, 10S)-3,12-di-hidroxicalameneno (25)<smiles>Cc1cc2c(cc1O)[C@@H](C)[C@H](O)C[C@@H]2C(C)CO</smiles>

(+)-(7S, 9R, 10S)-3,9,12-tri-hidroxicalameneno (24)<smiles>Cc1cc2c(C(C)CO)ccc(C)c2cc1O</smiles>

3,12-di-hidroxicadaleno (26)<smiles>Cc1cc2c(C(C)(O)CO)ccc(C)c2cc1O</smiles>

3,11,12-tri-hidroxicadaleno (27)

Figura 7. Sesquiterpenos cadalenos e calamenenos isolados de Phomopis cassiae

\subsection{Benzopiranos}

Os benzopiranos são substâncias formadas pela fusão de um anel benzênico com um anel heterociclo, e apresentam diversas atividades biológicas, como antimicrobiana, antimicobacteriana, entre outras. ${ }^{44,45}$

Curvularia sp., um endófito de Ocotea corymbosa (Lauraceae), foi cultivado em MBD e forneceu o extrato bruto de AcOEt que mostrou moderada atividade antifúngica contra C. cladosporioides e C. sphaerospermum. Fracionamento cromatográfico forneceu as benzopironas inéditas 5-metóxi-2-metil-benzopiran-4-ona (28) e 2,3-di-hidro-2-metil-5-metóxibenzopiran-4-ona (29) e as conhecidas (12S)- 2-(propan-12-ol)-5-hidroxi-benzopiran-4-ona (30) e 2,3-di-hidro-2-metil-benzopiran-4,5diol (31) (Figura 8). As substâncias 28-31 foram avaliadas nos ensaios com os fungos fitopatogênicos $C$. cladosporioides e $C$. sphaerospermum, somente $\mathbf{2 8}$ e $\mathbf{3 0}$ apresentaram fraca atividade com limite de detecção de $10 \mu \mathrm{g}$ para ambas as substâncias. Avaliação da atividade citotóxica utilizando HeLa (tumor de cervix humano) e CHO (ovário de hamster chinês) evidenciou que $\mathbf{3 0}$ reduziu a proliferação celular de HeLa em $70 \%$ e em $25 \%$ de células de $\mathrm{CHO}$ a uma concentração de $20 \mu \mathrm{mol} \mathrm{L}{ }^{-1}$. As substâncias 28, 29 e $\mathbf{3 1}$ foram inativas em $\mathrm{CHO}$ e apresentaram fraco efeito estimulatório (estimula a proliferação celular) em células HeLa. A atividade biológica de $\mathbf{2 8}$ e $\mathbf{3 0}$ parece ser diferenciada em células de fungos e 
mamíferos e também diferente para células normais de mamíferos ( $\mathrm{CHO}$ ), indicando um possível mecanismo de ação específico, o que merece futuras investigações. ${ }^{45}$<smiles>COc1cccc2oc(C)cc(=O)c12</smiles>

5-metoxi-2-metil-benzopiran-4-ona (28)<smiles>COc1cccc2c1C(=O)CC(C)O2</smiles>

2,3-di-hidro-2-metil-5-metoxi-benzopiran-4-ona (29)<smiles>CC(O)Cc1cc(=O)c2c(O)cccc2o1</smiles><smiles>C[C@@H]1C[C@H](O)c2c(O)cccc2O1</smiles>

(12S)-2-(propan-12-ol)-5-hidroxi-benzopiran-4-ona

(30) 2,3-di-hidro-2-metil-benzopiran-4,5-diol (31)

Figura 8. Benzopiranos isolados de Curvularia sp.

\subsection{Tetralonas}

Cryptocaria mandioccana Nees foi submetida à metodologia convencional para isolamento dos endófitos associados. Dos 11 fungos isolados, Colletotrichum gloeosporioides foi cultivado em MBD e o extrato bruto em AcOEt obtido forneceu (-)cis-4-hidroxi-6-deoxiscitalona (32) e (4R)-4,8di-hidroxi- $\alpha$-tetralona (33) (Figura 9). Estas substâncias foram ensaiadas in vitro contra os fungos fitopatogênicos $C$. cladosporioides e C. sphaerospermum e mostraram forte atividade com limite de detecção de 5,0 $\mu \mathrm{g}$, comparável ao padrão nistatina. ${ }^{46}$<smiles>O=C1C[C@H](O)[C@H](O)c2cccc(O)c21</smiles>

cis-4-hidroxi-6-deoxiscitalona (32)<smiles>O=C1CC[C@H](O)c2cccc(O)c21</smiles>

(4R)-4,8-di-hidroxi- $\alpha$-tetralona

Figura 9. Tetralonas isoladas de Cryptocaria mandioccana Nees

\subsection{Outras substâncias}

O extrato bruto em AcOEt obtido de Xylaria sp., um endófito de Palicourea marcgravii, apresentou forte atividade contra os fungos fitopatogênicos $C$. cladosporioides e C. sphaerospermum. O Fracionamento cromatográfico deste extrato utilizando coluna de sílica gel C-18 e CLAE (Cromatografia Líquida de Alta Eficiência) conduziu ao isolamento de griseofulvina (34), 7-declorogriseofulvina (35) e do ácido 2-hexil- 3-metil-butanodioico (36) (Figura 10).

Griseofulvina (34) é utilizada como um poderoso antimicótico, sendo comercializada em medicamentos como Fulcin ${ }^{\hat{a}}$ (Zeneca) e Sporostatinâ (Shering Plough) com atividade contra várias espécies de Microsporum, Epidermophyton e Trychophyton. $\mathrm{O}$ ácido 2hexilideno-3-metilbutanodioico mostrou-se ativo frente às linhagens de $C$. cladosporioides e $C$. sphaerospermum com limite de detecção de 10 e $25 \mu \mathrm{g}$, respectivamente. $^{35}$ 
De Periconia atropurpurea, um endófito de Xylopia aromatica, foram obtidas a cumarina (37), o benzaldeído (38) inéditos, e a periconicina (39) (Figura 10). As substâncias 37-39 foram submetidas à bioensaios com duas linhagens celulares de mamíferos HeLa e CHO. A substância $\mathbf{3 7}$ mostrou-se inativa nas duas linhagens celulares, e $\mathbf{3 8}$ induziu a proliferação de HeLa em $37 \%$ a $2,0 \mu \mathrm{mol} \mathrm{L}^{-1} \mathrm{e}$ de $\mathrm{CHO}$ em $38 \%$ a $20,0 \mu \mathrm{mol} \mathrm{L}{ }^{-1}$. A substância 39 diminuiu a viabilidade celular de HeLa e CHO com $\mathrm{Cl}_{50} 8,0 \mu \mathrm{mol} \mathrm{L}^{-1}$ mostrando potencial similar ao da cisplatina, um agente antineoplásico com $\mathrm{Cl}_{50} 5,0 \mu \mathrm{mol} \mathrm{L^{-1 }}$, o qual foi utilizado como controle positivo citotóxico. $^{47}$

Ensaios com os fungos fitopatogênicos $C$. cladosporioides e C. sphaerospermum revelaram que 38 possui potente atividade antifúngica com limite de detecção de $1,0 \mu \mathrm{g}$, comparável com a nistatina, usada como controle positivo. Já as substâncias 37 e 39 mostraram-se inativas. ${ }^{47}$

Phomopsis cassiae, um endófito de Cassia spectabilis foi submetido a estudo químico e forneceu três substâncias, 2,4-di-hidróxi-5,6dimetil benzoato de etila (40), phomopsilactona (41) inéditas, e o ácido hidroxifenil acético (42). As substâncias 40-42 mostraram forte atividade antifúngica contra fungos fitopatogênicos $C$. cladosporioides e $C$. sphaerospermum com limite de detecção de 1,0 $\mu \mathrm{g}$. As substâncias $\mathbf{4 0 - 4 2}$ foram testadas contra HeLa utilizando o teste MTT (ensaio colorimétrico baseado na conversão do sal 3(4,5-dimetil-2-tiazol)-2,5-difenil-2-H-brometo de tetrazolio em azul de formazana $)^{48}$. 0 composto 41 apresentou uma fraca citotoxicidade $\left(\mathrm{Cl}_{50} 200 \mu \mathrm{mol} \mathrm{L}^{-1}\right)$ e $\mathbf{4 2}$ uma forte citotoxicidade com $\mathrm{Cl}_{50} 10 \mu \mathrm{mol} \mathrm{L} \mathrm{L}^{-1}$. A cisplatina foi novamente usada como controle positivo. $^{49}$<smiles>[R]c1c(OC)cc(OC)c2c1O[C@]1(C(=O)[C@@]2(O)OC)C(C)=CC(=O)C[C@H]1[CH]</smiles>

$\mathbf{R}=\mathbf{H}$; griseofulvina (34)

$\mathbf{R}=\mathbf{C l}$; 7-declorogriseofulvina (35)<smiles>CCCCCC=C(C(=O)O)C(C)C(=O)O</smiles>

ácido 2-hexil-3-metil-butanodioico (36)<smiles>CCC(=O)Cc1cc2cc(OC)cc(OC)c2oc1=O</smiles>

6,8-dimetoxi-3-(2'-oxo-propil) -cumarina (37)<smiles>CCOC(=O)c1c(O)cc(O)c(C)c1C</smiles><smiles>C/C=C/C=C/c1cc(O)cc(O)c1C=O</smiles>

2,4-di-hidroxi-6-((1'E,3'E)-penta1',3'-dienil)benzaldeído (38)<smiles>C=C1OC(=O)c2c(O)c(C)c(O)c(C=O)c2C1C</smiles><smiles>CC(CO)C1=C2CC[C@]3(C)CC4C(C(=O)[C@H](O)[C@@H]4C)C(C=O)C=CCC3[C@H]2C1</smiles>

periconicina (39)<smiles>O=C(O)Cc1ccccc1O</smiles>

2,4-di-hidroxi-5,6-dimetil benzoato de etila (40) phomopsilactona (41) ácido hidroxifenil acético (42) 


\section{Conclusões}

Dez anos de pesquisas envolvendo microorganismos endofíticos trouxe ao nosso grupo uma experiência fantástica em termos de conhecimento do metaboloma de endófitos, das substâncias inéditas e bioativas produzidas por micro-organismos isolados de espécies vegetais endêmicas da flora brasileira. Nossa coleção de fungos atinge o número de 200 endófitos, sendo que 45 já estão classificados por taxonomia convencional ou biologia molecular. As espécies predominantes observadas, Xylaria, Colletotrichum e Phomopsis, produzem as mais diversas classes de substâncias.

Das quarenta e duas substâncias apresentadas neste trabalho, todas apresentam algum tipo de atividade biológica e $42 \%$ são estruturas inéditas. Para as substâncias conhecidas, já haviam sido relatadas inúmeras atividades biológicas, das inéditas todas apresentaram atividade nos ensaios testados em nosso grupo de pesquisa, contra os fungos fitopatogênicos $C$. cladosporioides e C. sphaerospermum, citotóxica nas linhagens HeLa e CHO, inibitória da enzima acetilcolinesterase e das enzimas subtilisina e tripsina, sendo que em pelo menos uma destas as substâncias inéditas são ativas. Deste modo, verifica-se o enorme potencial destes micro-organismos, os quais podem, no futuro, ter aplicação na medicina, agricultura ou indústria.

Estes resultados refletem a potencialidade dos endófitos na produção de substâncias inéditas e bioativas e mostram a urgente necessidade do estudo da microbiota associada às espécies vegetais da flora brasileira, tendo em vista o risco da perda da biodiversidade endofítica, devido à rápida diminuição das florestas tropicais.

\section{Agradecimentos}

À CAPES, CNPq e FAPESP (Biota, BIOprospecTA) pelas bolsas concedidas e pelo apoio financeiro à pesquisa.

\section{Referências Bibliográficas}

${ }^{1}$ Butler, M. S. J. Nat. Prod. 2004, 67, 2141. [CrossRef] [PubMed]

2 Tulp, M.; Bohlin, L. Drug Discov. Today 2004, 9, 450. [CrossRef] [PubMed]

${ }^{3}$ Strobel, G.; Daisy, B. Microbiol. Mol. Biol. Rev. 2003, 67, 491. [CrossRef] [PubMed]

${ }^{4}$ Strobel, G.; Daisy, B.; Castillo, U.; Harper, J. J. Nat. Prod. 2004, 67, 257. [CrossRef] [PubMed]

${ }^{5}$ Cragg, G. M.; Newman, J. D. Pure Appl. Chem. 2005, 77, 7. [CrossRef]

${ }^{6}$ Greve, H.; Mohamed, I. E.; Pontius, A.; Kehraus, S.; Gross, H.; Konig, G. M. Phytochem. Rev. 2010, 9, 537. [CrossRef]

${ }^{7}$ Guo, B.; Wang, Y.; Sun, X.; Tang, K. Appl. Biochem. Microbiol. 2008, 44, 136. [CrossRef] [PubMed]

${ }^{8}$ Newman, D. J.; Cragg, G. M. J. Nat. Prod. 2012, 75, 311. [CrossRef] [PubMed]

${ }^{9}$ Newman, D. J.; Cragg, G. M. J. Nat. Prod. 2007, 70, 461. [CrossRef] [PubMed]

${ }^{10}$ Carter, G. T. Nat. Prod. Rep. 2011, 28, 1783. [CrossRef] [PubMed]

${ }^{11}$ Esposito, E.; Azevedo, J. L.; Fungos uma introdução a biologia, bioquímica $e$ biotecnologia, 2a. ed., EDUCS: Caxias do Sul, 2010.

${ }^{12}$ Kharwar, R. N.; Mishra, A.; Gond, S. K.; Stierle, A.; Stierle, D. Nat. Prod. Rep. 2011, 28, 1208. [CrossRef] [PubMed]

${ }^{13}$ Jalgaonwala, R. B.; Mohite, B. V.; Mahajan, R. T. J. Microbiol. Biotech. Res. 2011, 1, 21. [Link]

${ }^{14}$ Arnold, A. E. Fungal Biol. Rev. 2007, 21, 51. [CrossRef]

${ }^{15}$ Tan, R. X. Zou, W. X. Nat. Prod. Rep. 2001, 18, 448. [CrossRef] [PubMed]

${ }^{16}$ Melo, I. S.; Azevedo, J. L.; Ecologia Microbiana, Embrapa-CNPMA: Jaguariúna, 1998. 
${ }^{17}$ Aly, A. H.; Debbad, A.; J.; Proksch, P. Appl. Microbiol. Biotechnol. 2011, 90, 1829. [CrossRef] [PubMed]

${ }^{18}$ Aly, A. H.; Debbad, A.; Kjer, J.; Proksch, P. Fungal Divers. 2010, 41, 1. [CrossRef]

${ }^{19}$ Gunguli, B. N.; Deshmukh, S. K. Em Fungi Multifaceted Microbes; Azevedo, J. L.; Araujo, W. L., eds.; Taylor \& Francis: London, 2007, cap 12.

${ }^{20}$ Silva, G. H.; Oliveira, C. M.; Teles, H. L.; Bolzani, V. S.; Araujo, A. R.; Pfenning, L. H.; Young, M. C. M.; Costa-Neto, C. M.; Haddad, R.; Eberlin, M. N. Quim. Nova 2010, 33, 2038. [CrossRef]

${ }^{21}$ Gunatilaka, A. A. L. J. Nat.Prod. 2006, 69, 509. [CrossRef] [PubMed]

${ }^{22}$ Araújo, W. L.; Lacava, P. T.; Marcon, J.; Lima, A. O. S.; Sobral, J. K.; Pizzirani-Kleiner, A. A.; Azevedo, J. L.; Guia Pratico: Isolamento e Caracterização de Microrganismos Endofíticos, CALO: Piracicaba, 2010.

${ }^{23}$ Ownley, B. H.; Gwinn, K. D.; Vega, F. E. BioControl 2010, 55, 113. [CrossRef]

${ }^{24}$ Paranagama, P. A.; Wijerante, E. M. K.; Gunatilaka, A. A. L. J. Nat. Prod. 2007, 70, 1939. [CrossRef] [PubMed]

${ }^{25}$ Yang, X.; Zhang, J.; Luo, D. Nat. Prod. Rep. 2012, 29, 622. [CrossRef] [PubMed]

${ }^{26}$ Schulz, B.; Boyle, C.; Braeger, S.; Rommert, A.; Krohn, K. Mycol. Res. 2002, 106, 996. [CrossRef]

${ }^{27}$ Wang, L.; Xu, B.; Su, Z.; Lin, F.; Zhang, C.; Kubicek, C. P. Appl. Microbiol. Biotechnol. 2012, 93, 1231. [CrossRef] [PubMed]

${ }^{28}$ Ondeyka, J. G.; Helms, G. L.; Hensens, O.D.; Goetz, M. A.; Zink, D. L.; Tsipouras, A.; Shoop, W. L.; Slayton, L.; Dombrowski, A. W.; Polishook, J. D.; Ostlind, D. A.; Tsou, N. N.; Ball, R. G.; Singh, S. B. J. Am. Chem. Soc. 1997, 119, 8809. [CrossRef]

${ }^{29}$ Zhang, B.; Salituro, G.; Szalkowski, D.; Li, Z.; Zhang, Y. Royo, I.; Vilella, D.; Díez, M. T.; Pelaez, F.; Ruby, C.; Kendall, R. L.; Mao, X.; Griffin, P.; Calaycay, J.; Zierath, J. R.; Heck, J. V.; Smith, R. G.; Moller, D. E. Science 1999, 284, 974. [CrossRef] [PubMed]

${ }^{30}$ Guo, B.; Dai, J.; Ng, S.; Huang, Y.; Leong, C.; Ong, W.; Carte, B. K. J. Nat. Prod. 2000, 63, 602. [CrossRef] [PubMed]
${ }^{31}$ Zhang, H. W.; Song, Y. C.; Tan, R. X. Nat. Prod. Rep. 2006, 23, 753. [CrossRef] [PubMed]

${ }^{32}$ Shweta, S.; Zuehlke, S.; Ramesha, B. T.; Priti, V.; Mohana, K. P.; Ravikanth, G.; Spiteller, M.; Vasudeva, R. Phytochemistry 2010, 71, 117. [CrossRef] [PubMed]

${ }^{33}$ Kusari, S.; Spiteller, M. Nat. Prod. Rep. 2011, 28, 1203. [CrossRef] [PubMed]

${ }^{34}$ Melissa M. Wagenaar, M. M.; Corwin, J.; Strobel, G.; Clardy, J. J. Nat. Prod. 2000, 63, 1692. [CrossRef] [PubMed]

${ }^{35}$ Cafêu, M. C; Silva, G. H.; Teles, H. L.; Bolzani, V. S.; Araujo, A. R.; Young, M. C. M.; Pfenning, L. H. Quim. Nova, 2005, 28, 991. [CrossRef]

${ }^{36}$ Guimarães, K. L., Souza-Filho, J. D., MaresGuia, T. R., Braga, F. C. Phytochemistry 2008, 69, 439; [CrossRef] Krohn, K., Bahramsari, R., Flörke, U., Ludewig, K., Kliche-Spory, C., Michel, A., Qust, H. -J., Draeger, S., Schulz, B., Antus, S. Phytochemistry 1997, 45, 313; [CrossRef] Kuramata, M., Fujioka, S., Shimada, A., Kawano, T., Kimura, Y. Biosci. Biotech. Biochem. 2007, 71, 499; [Link] Özcan, S., Sahin, E., Balci, M. Tetrahedron Lett. 2007, 48, 2151. [CrossRef]

${ }^{37}$ Oliveira, C. M.; Silva, G. H.; Regasini, L. O.; Zanardi, L. M.; Evangelista, A. H.; Young, M. C. M.; Bolzani, V. S.; Araujo, A. R. Z. Naturforsch., C: J. Biosci. 2009, 64c, 824. [Link]

${ }^{38}$ Oliveira, C. M.; Regasini, L. O.; Silva, G. H.; Pfenning, L. H.; Young, M. C. M.; Berlink, R. G. S.; Bolzani, V. S.; Araujo, A. R. Phytochemistry Lett. 2011, 4, 93. [CrossRef]

${ }^{39}$ McDonald, L. A.; Barbieri, L. R.; Bernan, V. S.; Janso, J.; Lassota, P.; Carter, G. T. J. Nat. Prod. 2004, 67, 1565. [CrossRef] [PubMed]

${ }^{40}$ Silva, G. H.; Oliveira, C. M.; Teles, H. L.; Pauletti, P. M.; Castro-Gamboa, I.; Silva, S. H. S.; Bolzani, V. S.; Young, M. C. M.; CostaNeto, C. M.; Pfenning, L. H.; Berlink, R. G. S.; Araujo, A. R. Phytochemistry Lett. 2010, 3, 164. [CrossRef]

${ }^{41}$ Oliveira, C. M.; Silva, G. H.; Regasini, L. O.; Flausino Jr., O.; López, S. N.; Abissi, M.; Berlink, R. G. S.; Sette, L. D.; Bonugli-Santos, R. C.; Rodrigues, A.; Bolzani, A. S.; Araujo, A. 
R. J. Nat. Prod. 2011, 74, 1353. [CrossRef] [PubMed]

${ }^{42}$ Li, G.; Duan, M.; Yu, Z.; Li, L.; Dong, J.; Wang, X.; Guo, J.; Huang, R.; Wang, M.; Zhang, K. Phytochemistry 2008, 69, 1439; [CrossRef] Hiramatsu, F.; Murayama, T.; Koseki, T.; Funakoshi, T.; Shiono, Y. Nat. Prod. Res. 2011, 25, 781. [CrossRef]

${ }^{43}$ Silva, G. H.; Teles, H. L.; Zanardi, L. M.; Young, M. C. M.; Eberlin, M. N.; Haddad, R.; Pfenning, L. H.; Costa-Neto, C. M.; CastroGamboa, I.; Bolzani, V. S.; Araujo, A. R. Phytochemistry 2006, 67, 1964. [CrossRef] [PubMed]

${ }^{44}$ Bungihan, M. E.; Tan, M. A.; Kitajima, M.; Kogure, N.; Franzblau, S. C.; Cruz, T. E. E.; Takayama, H.; Nonato, M. G. J. Nat. Med. 2011, 65, 606; [CrossRef] Wu, Z.; Li, D.; Chen, Y.; Zhang, W. Helv. Chim. Acta 2010, 93, 920; [CrossRef] Tripathi, R. P.; Bisht, S. S.; Pandey, V. P.; Pandey, S. K.; Singh, S.; Sinha, S. K.; Chaturvedi, V. Med. Chem. Res. 2011, 20, 1515. [CrossRef]
${ }^{45}$ Teles, H. L.; Silva, G. H.; Castro-Gamboa, I.; Bolzani, V. S.; Pereira, J. O.; Costa-Neto, C. M.; Haddad, R.; Eberlin, M. N.; Young, M. C. M.; Araujo, A. R. Phytochemistry 2005, 66, 2363. [CrossRef] [PubMed]

${ }^{46}$ Inácio, M. L.; Silva, G. H.; Teles, H. L.; Trevisan, H. C.; Cavalheiro, A. J.; Bolzani, V. S.; Young, M. C. M.; Pfenning, L. H.; Araujo, A. R. Biochem. Syst. Ecol. 2006, 34, 822. [CrossRef] ${ }^{47}$ Teles, H. L.; Sordi, R.; Silva, H. G.; CastroGamboa, I.; Bolzani, V. S.; Pfenning, L. H.; Abreu, L. M.; Costa-Neto, C. M.; Young, M. C. M.; Araujo, A. R. Phytochemistry 2006, 67, 2686. [CrossRef] [PubMed]

${ }^{48}$ Mosmann, T. J. Immunol. Methods 1983, 65, 55. [CrossRef]

${ }^{49}$ Silva, G. H.; Teles, H. L.; Trevisan, H. C.; Bolzani, V. S.; Young, M. C. M.; Pfenning, L. H.; Eberlin, M. N.; Haddad, R.; Costa-Neto, C. M.; Araujo, A. R. J. Braz. Chem. Soc. 2005, 16, 1463. [CrossRef] 